\title{
Gestión estratégica de operaciones en empresas mixtas
}

\section{Strategic operations management in mixed companies}

\section{Leonardo Arteaga}

arteagaleon30@gmail.com

https://orcid.org/0000-0002-4200-5024

Ministerio de Petróleo Venezuela, Costa Sur del Lago Trujillo - Venezuela
Artículo recibido en febrero 2021

Arbitrado en marzo 2021

Aceptado en mayo 2021

Publicado en julio 2021

La investigación tuvo como objetivo analizar la gestión estratégica de operaciones en las empresas mixtas de la industria petrolera venezolana. El estudio es descriptivo, con diseño no experimental, transeccional, y de campo. La población quedó conformada por las empresas mixtas de la industria petrolera venezolana en la Costa Sur del Lago-Trujillo. Los sujetos informantes fueron los gerentes generales, de operaciones de producción y de operaciones de mantenimiento. Se aplicó la encuesta, con un cuestionario de 57 ítems, validado mediante el juicio de expertos. La confiabilidad se calculó a través del coeficiente Alfa de Cronbach, obteniéndose 0,9321 . El análisis de los datos se realizó mediante la estadística descriptiva, a través de las frecuencias y media aritmética. Se concluyó que estas empresas, ostentan excelente gestión estratégica de operaciones, a través del proceso de operaciones, capacidad del proceso de operaciones, proceso de gestión estratégica de operaciones y de las estrategias de operaciones.

Palabras clave: Proceso; Estrategia; Gestión estratégica; Empresa mixta; Industria; Operaciones

The research aimed to analyze the strategic management of operations in the mixed companies of the Venezuelan oil industry. The study is descriptive, with non-experimental, transectional, and field design. The population was formed by the mixed companies of the Venezuelan oil industry on the South Coast of Lake-Trujillo. The reporting subjects were general managers, production operations and maintenance operations. The survey was applied, with a 57-item questionnaire, validated through expert judgment. Reliability was calculated through Cronbach's alpha coefficient, obtaining 0.9321. Data analysis was performed using descriptive statistics, through frequencies and arithmetic mean. It is concluded that these companies have excellent strategic management of operations, through the fulfillment of the operations process, capacity of the operations process, strategic operations management process, and operations strategies.

Keywords: Process; Strategy; Strategic management; JV; Industry; Operations 
INTRODUCCION

En la actualidad, la situación de las empresas no es tan distinta a las localidades del planeta: encontrándose con un mercado altamente competitivo, globalizado, con una nueva visión para hacer negocios, con una tecnología que avanza vertiginosamente y una brecha significativa entre sus competidores, sumado a ello, un entorno macroeconómico de gran incertidumbre.

Dentro de este contexto, las empresas necesitan estar a la vanguardia en el conocimiento de herramientas gerenciales, que les permitan mejorar su competitividad y desempeño basado en la toma de decisiones. Por ello, es importante determinar si las estrategias formuladas satisfacen las expectativas de rendimiento.

En este sentido, se considera que debe existir un compromiso de los entes involucrados en las actividades para que haya una perfecta coordinación entre los procesos y las estrategias. Desde esta perspectiva, la industria petrolera venezolana, según López (2008), ha internalizado sus procesos como fundamento para el desarrollo de una gestión estratégica, llevando a cabo actividades que incentiven el crecimiento sostenido del sector, impulsado desde una visión corporativa, en pro de la búsqueda de ventajas competitivas perdurables; pero esto no ha sido suficiente para lograr su cometido.

Por otro lado, los continuos procesos de transformación demandados por la globalización creciente de la economía, el acelerado desarrollo tecnológico, la innovación, la introducción de nuevos productos en el mercado, y las necesidades cambiantes de los clientes, han modificado la realidad del sector petrolero a nivel mundial, exigiéndole a estas empresas, que son el motor de muchas economías, revisar la forma de llevar sus operaciones y formular estrategias para adaptarse exitosamente al nuevo entorno competitivo, propiciando una evolución en el mercado.

Con relación a lo anterior, a criterio de López
(2008), en el caso venezolano, la situación de crisis económica generalizada actual, ha obligado a que las organizaciones, tanto públicas como privadas, se transformen y tengan una visión más articulada en su conjunto, abriendo espacio para la concertación en cuanto a la modificación de los enfoques y supuestos del paradigma empresarial convencional en búsqueda de ventajas competitivas. Este hecho ha llevado a considerar nuevas estrategias en todos los procesos que ejecutan.

Dentro de este orden de ideas, resulta imprescindible prepararse para lo que vendrá y así estar listo para los competidores, nuevos clientes, mercados y retos. Uno de estos nuevos retos lo representa el aumento de la competitividad empresarial; por ello un gerente debe tener siempre enmarcada sus ideas y pensamientos dentro de la gestión estratégica de operaciones.

De allí que, la gestión estratégica de operaciones en las organizaciones productivas $\mathrm{o}$ manufactureras, motivado a la trascendencia que tienen, sea una herramienta clave para el alcance de la competitividad, el desarrollo económico de un país y el mejoramiento de la calidad de vida de sus habitantes, así como el valor de sobrevivencia en el tiempo dentro del sistema de globalización mundial.

Es por ello que aparece la estrategia de operaciones, la cual, según Las Heras (2008), es una visión de la función de operaciones que se debe integrar con la estrategia empresarial y, debe dar como resultado un patrón consistente de toma de decisiones de operaciones y una ventaja competitiva para la compañía.

En este sentido, la estrategia de operaciones no se puede formar en el vacío, ya que afecta, y es afectada, por grupos dentro y fuera de ella. No puede ser definida solo desde la perspectiva de los que trabajan en operaciones, sino desde la perspectiva superior de la organización involucrando las áreas restantes. 
Es precisamente por estas razones que las empresas requieren, además de formular e implementar estrategias, ejercer una gerencia estratégica de sus operaciones logrando obtener elementos diferenciadores en sus productos o servicios, es decir, competencias distintas que permiten a un cliente particular seleccionar una compañía y no otras.

De lo anterior se deduce que, las empresas independientemente de la naturaleza de su negocio se deben monitorear y estar alerta antes señales de debilidad emitidas por la organización. Más aún, si estas empresas poseen un cliente exigente en cuanto a competencias distintivas dentro del sector donde se encuentra inmersa esta compañía, como es el caso bajo estudio.

En este contexto, Krajewski y Ritzman (2008) revelan que la gestión estratégica de operaciones es decisiva para cada tipo de organización, porque esta sólo puede alcanzar sus metas mediante la acertada dirección de personas, capital, información y materiales. Hoy, el término gestión estratégica de operaciones se refiere a la dirección, así como el control de los procesos mediante los cuales los insumos se transforman en servicios en las organizaciones.

De lo expuesto, se comprende que trabajar efectivamente con otras áreas funcionales de la organización es una de las responsabilidades más importantes dela gestión estratégica de operaciones, razón por la cual es fundamental a nivel gerencial que los límites funcionales no escondan la eficiencia en las operaciones, si no que más bien las funciones estén correctamente delimitadas y alineadas con la estrategia corporativa.

Esta se debe basar en la misión de la organización, en esencia reflejar la forma en la cual se utilizan los recursos en las diferentes áreas funcionales, tales como finanzas, mercadotecnia y operaciones, para logar la ventaja competitiva. Es decir, la estrategia de operaciones específica la manera en que la empresa empleará sus capacidades de producción o servicio para apoyar la estrategia organizacional.

En este marco contextual, Skinner (2010) afirma que, una dirección estratégica de operaciones bien establecida supone el pilar directivo fundamental para directivos y empleados, quienes serán los responsables de llevarla a cabo; al mismo tiempo debe responder al grupo de intereses externos con los cuales interactúa, constituyéndose en el plan de acción. Al respecto, Slack y otros (2007), exponen que la implantación de la estrategia implica un patrón de decisiones, así como acciones destinadas a la consecución de un plan.

Los autores recalcan que, los gerentes de operaciones deben estar conscientes que para la toma de decisiones estratégicas se deben analizar puntualmente como se realiza el proceso de operaciones, cual es la capacidad de su proceso de operaciones, y como se aplica el proceso de gestión estratégica de operaciones en su organización.

Ahora bien, en el caso de América Latina, según López (2008), la gestión estratégica de operaciones de las empresas se prepara para prever los impactos gerenciales, así como también los acondicionamientos económicos, políticos, sociales y tecnológicos, producto de la intervención o las variaciones en escenarios diversos.

Las empresas bajo estos escenarios, en su afán de responder a estos impactos, y sumados a las exigencias tanto del mercado como las del consumidor, procuran desarrollar productos/ servicios de calidad como alternativa para ser competitivas. De esta manera, en el contexto empresarial surge la concepción de gestión estratégica de operaciones como una herramienta competitiva, que soporta a las empresas para optimizar sus recursos generando valor en sus procesos cumpliendo con las entregas al consumidor final con altos niveles de satisfacción (Skinner, 2010).

Planteadas las ideas sobre las definiciones de gestión estratégica de operaciones, su importancia, 
aplicabilidad y aspectos a considerar en su estudio, a continuación, se hace referencia al sector que representa la inquietud del investigador. En este contexto, hoy día las organizaciones petroleras deben ser competitivas $y$ ofrecer entregas oportunas de productos de calidad, cuyo entorno de exigencias ha comprometido a gerentes y a ingenieros a optimizar todos sus procesos (López, 2008).

En esta perspectiva, al sector petrolero a nivel mundial se le confiere el carácter de base energética fundamental de la sociedad con base en el petróleo como fuente de energía con múltiple utilización, por ello la industria petrolera es considerada una de las más importantes.

Adentrando un poco más en el desarrollo del estudio, tal y como lo afirma López (2008), desde el último tercio del siglo XIX, prácticamente, todas las actividades económicas se sustentan en el petróleo como fuente energética, representando alrededor del $40 \%$ de la necesidad mundial, dándole así la importancia a la complejidad y aumento del mercado a través de un bien, con características intrínsecas, vinculadas con el acontecer de todo el planeta, como la industria petrolera.

Visto en esta perspectiva, la industria petrolera venezolana no debería quedar relegada del resto del mundo. En este sentido, Petróleos de Venezuela Sociedad Anónima (PDVSA), es la industria venezolana en el sector de hidrocarburos, que tiene como misión diseñar estrategias que permitan la recuperación eficiente y rentable para la reserva de este.

En específico, PDVSA es una corporación mundial de energía, propiedad del estado venezolano, que se encarga del desarrollo de la industria petrolera, petroquímica y carbonífera. Tiene como función principal planificar, coordinar, supervisar, controlar las actividades de sus empresas operadoras más las filiales, tanto en Venezuela como en el exterior.

Cabe resaltar, a efectos del trabajo que se desarrolla, que dentro de su estructura organizacional se ubican las empresas mixtas a través de convenios operativos, las cuales tienen como objetivo social el desarrollo de actividades de explotación en los campos petroleros cedidos bajo concesiones por el estado Venezolano, lo que involucra extracción en estado natural, recolección, transporte y almacenamiento inicial, además de prestar servicio de ingeniería, construcción, reconstrucción y reparación para el desarrollo de proyectos vinculados con Costa Afuera.

Estas empresas, según la observación directa del investigador, emplean un proceso de trabajo normalizado, basado en las mejores prácticas, guiadas por las normas y procedimientos creadas por ellas mismas, las cuales contienen lineamientos prácticos para la efectiva ejecución de sus operaciones, de manera sistemática u ordenada, tratando de no omitir ningún paso importante.

De esta manera se garantizan un alto grado de confianza en el éxito, así como en el cumplimiento de los requisitos exigidos por PDVSA. Éste, es el enfoque de las empresas mixtas, utilizado a fin de alcanzar las más altas metas de eficiencia dentro de un mundo totalmente cambiante como el actual.

Sin embargo, aun cuando en estas empresas se ha venido observando un mayor reconocimiento de la importancia que tiene lograr una efectiva gestión de operaciones para alcanzar un control eficiente del proceso operacional, según la percepción del personal que labora en la misma, existen deficiencias en la visión global de los procesos operacionales, lo cual ha generado debilidades en todo el proceso, ya que tanto la información como las operaciones no fluyen correctamente.

En específico, en entrevistas realizadas a los gerentes generales de estas empresas, se señalan la falta de herramientas gerenciales existente para la administración de las operaciones, repercutiendo de forma negativa en su operacionalidad. Asimismo, afirmaron que las operaciones se están viendo afectadas por fallas en la manera de ejecutar los procesos operacionales. 
Por otro lado, al entrevistar a los gerentes de operaciones acotaron que las fallas pudiesen estar enfocadas en la aplicación de una gerencia de operaciones tradicional, no así estratégica, develándose en falta de previsión de su capacidad operativa y de la escasa aplicación de procesos estratégicos de operaciones, esto último, según los entrevistados, por falta de una visión realista de las estrategias de operaciones que se están aplicando, lo que conlleva a deficiencias en la gestión de las operaciones.

Tomando en consideración los señalamientos planteados, y haciendo uso de la técnica de observación directa por parte del autor, se detectan algunos indicadores que reflejan una inadecuada gestión estratégica de operaciones en estas empresas.

Es de ahí que nace la imperante necesidad de que las líneas gerenciales de las mismas migren de la gestión operativa a la gestión estratégica, lo cual se evidencia en las entrevistas realizadas a través de: (a) poca vinculación a la teoría de la gestión estratégica de operaciones en el desarrollo o ejecución de los procesos operativos, (b) problemas con las entradas y salidas de los procesos, (c) falta de previsión de su capacidad operativa, (d) carencia de estrategias definidas y realistas en cuanto a gestión de operaciones.

\section{Gestión estratégica de operaciones}

A nivel estratégico el objetivo de la gestión de operaciones es participar en la búsqueda de una ventaja competitiva sustentable para la empresa. Por su parte, Chase y otros (2009), describen la gestión de operaciones como el diseño, la operación y la mejora de los sistemas que crean y entregan los principales productos y servicios de la empresa. Fernández y otros (2003), definen la gestión de operaciones como el estudio de la toma de decisiones en la función de operaciones.

Según Muñoz (2009), la gestión estratégica de operaciones es la guía, conducción y control de los esfuerzos de un grupo de individuos hacia un objetivo común, es el proceso que permite optimizar la función de producción, aumentar la calidad, mejorar la satisfacción de los clientes y disminuir los costos, fundamentado en la planificación para determinar qué se va a hacer, la toma de decisiones que incluyen el esclarecimiento de objetivos, establecimiento de políticas, fijación de programas-campañas, determinación de métodos o procedimientos específicos y fijación de previsiones.

Mientras que, Betancourt (2002) plantea que la estrategia de operaciones debe dar como resultado un patrón consistente de toma de decisiones y una ventaja competitiva para la compañía. Según este autor, la estrategia de operaciones es de tipo funcional, debe guiarse por la estrategia empresarial y dar como resultado un patrón consistente en la toma de decisiones que permita la adecuada gestión de las actividades de: diseño de bienes o servicios, calidad, procesos, recursos humanos, diseño del trabajo, localización, organización, abastecimiento, inventario, programación y mantenimiento.

Coincidiendo parcialmente con este planteamiento, Schroeder (2007), plantea que la gestión estratégica de operaciones tiene la responsabilidad de cinco importantes áreas de decisiones: proceso, capacidad, inventario, fuerza de trabajo y calidad. Mientras que Skinner (2010), expone que las decisiones más importantes relacionadas con esta gestión están relacionadas con la capacidad, ubicación, proceso y distribución física; proporcionando la dirección estratégica a largo plazo para la dirección de operaciones.

Se comprende entonces que, la gestión estratégica de operaciones proporciona una visión global en cuanto al tipo de empresa que la organización desea ser, guiando el conjunto de decisiones de planificación, organización, dirección y control que generen acciones presentes y futuras en las áreas de producción de bienes o servicios que comprende su diseño, procesos de 
trabajo, generación, calidad, así como actividades de localización, abastecimiento e inventario y mantenimiento, con la finalidad de generar ventajas competitivas sostenibles.

Bajo este enfoque, el investigador aborda el desglose de la variable gestión estratégica de operaciones en cuatro (4) aspectos o dimensiones: proceso de operaciones, capacidad del proceso, proceso de gestión estratégica y estrategias de operaciones; lo cual permitirá establecer conclusiones y recomendaciones validas que orienten la gestión estratégica de operaciones en las empresas mixtas seleccionadas.

\section{Proceso de operaciones}

De acuerdo a los señalamientos expresados por Arnoletto (2010), los procesos de operaciones están sujetos a la estrategia de operaciones que fije la empresa, la misma busca constituirse en un plan de acción a largo plazo para la producción de los bienes y servicios de la empresa, propendiendo al logro de los objetivos globales mediante decisiones estratégicas, tácticas y operativas congruentes. Abarca objetivos operativos de largo plazo, estrategias operativas, distribución de recursos entre los productos y/o funciones y criterios para tomar decisiones.

Por otro lado, el proceso de operaciones es el plan de acciones en cada área funcional para lograr los objetivos operativos; de tal forma de ayudar a la organización a lograr su misión en conjunto y crear una ventaja competitiva. Mientras que, la gestión estratégica es un proceso de evaluación sistemática de la naturaleza de un negocio, definiendo las necesidades a largo plazo, identificando metas y objetivos cuantitativos, desarrollando habilidades para alcanzar dichos objetivos y, localizando recursos para llevar a cabo las estrategias planteadas (Arnoletto, 2010).

De lo expuesto, se puede deducir que la gestión estratégica de operaciones está referida al proceso que se ocupa del establecimiento de políticas y planes amplios en los cuales pueden ser utilizados de forma óptima los recursos que se relacionan con el diseño, operaciones, al igual que la mejora de los sistemas relacionados con la producción de bienes y servicios de la empresa, con el fin de apoyar mejor su estrategia competitiva a largo plazo, para facilitar el logro de la misión (Arnoletto, 2010). Visto así, la gestión estratégica de operaciones debe considerar en un principio el proceso de operaciones como factor clave en el logro de sus objetivos.

Ahora bien, el proceso de operaciones es una visión de la función de operaciones, que depende de la dirección o impulso general para la toma de decisiones. Esta visión se debe integrar con la estrategia empresarial y con frecuencia, aunque no siempre, se refleja en un plan formal. El proceso de operaciones debe dar como resultado un patrón consistente de toma de decisiones y una ventaja competitiva para la compañía.

Bajo este enfoque, el proceso de operaciones como proceso en sí, tiene como finalidad determinar criterios y métodos para asegurar que las actividades que comprende dicho proceso se realicen de manera eficaz, al igual que el control de este. Esto implica, centrarse en actividades operacionales, así como en todas las características relevantes que permitan el control de las mismas y la gestión del proceso (Rodríguez, 2008).

Por otro lado, la descripción de las actividades de este proceso se consigue a través de un diagrama, donde se pueden representar estas actividades de manera gráfica e interrelacionadas entre sí (Rodríguez, 2008). Estos diagramas facilitan la interpretación de las actividades en su conjunto, debido a que se permite una percepción visual del flujo y secuencia de las mismas, incluyendo las entradas y salidas necesarias para el proceso y límites de este. De tal manera, que la decisión del tipo de procesos y selección de este son tema crucial para quien gestiona las operaciones.

Con base a lo expuesto, el investigador deduce que, en virtud de obtener la satisfacción total 
del cliente y lograr altos niveles de rendimiento financiero, es necesario analizar los procesos operacionales de la organización, con la finalidad de detectar las áreas críticas y poder aplicar los correctivos necesarios de forma oportuna. En la perspectiva del proceso de operaciones los directivos deben identificar los procesos críticos en los que deben sobresalir con excelencia, si es que quieren satisfacer los objetivos de los segmentos de accionistas y de clientes seleccionados.

\section{Capacidad del proceso de operaciones}

La capacidad del proceso es la medida de la reproducibilidad intrínseca del producto resultante de un proceso. Independientemente del modelo de proceso que se utilice, los directores de operaciones deben determinar la capacidad.

Esta decisión afecta a una gran parte del costo fijo, y también establece si se satisfará la demanda o si las instalaciones estarán inactivas. Si la planta es demasiado grande, parte de ella estará inactiva, con lo que se añade costo a la producción existente $y$, si la planta es demasiado pequeña, se pierden clientes. Así, el tamaño de la planta es fundamental para alcanzar altos niveles de utilidad.

Conforme a Krajewski y Ritzman (2008), la capacidad es la tasa de producción máxima de una instalación, que puede ser una estación de trabajo o una organización entera. El gerente de operaciones debe suministrar la capacidad necesaria para satisfacer la demanda actual y futura; de otra manera, la organización perdería oportunidades de crecimiento y de ganar beneficios.

De acuerdo a lo descrito, para el investigador el término capacidad implica un ritmo alcanzable de producción; por ejemplo, un número de unidades por día, puede no decir nada respecto al tiempo que puede sostenerse este ritmo, porque podrían representar un máximo de un día o un promedio de seis meses, para evitar este problema se emplea, según lo acotan Chase y otros (2009), el concepto de nivel óptimo de operación, que representa el nivel de capacidad para el cual el proceso fue diseñado y, por consiguiente el volumen de producción al que se minimiza el costo unitario promedio.

\section{Proceso de gestión estratégica de operaciones}

La gerencia estratégica es un proceso gerencial debidamente planificado, en el cual se toman un conjunto de decisiones interactivas y superpuestas conducentes al desarrollo de una estrategia eficaz para la empresa. Coincidiendo con este planteamiento, Sallenave (2009) señala que, el proceso de gestión estratégica de operaciones puede resumirse en una secuencia de pasos, los cuales pueden ayudar a preparar el análisis de caso de política empresarial.

Los pasos mencionados por Sallenave (2009), son agrupados por David (2008), en tres (3) etapas, siendo éstas: formulación, ejecución y evaluación estratégica; quien señala que la formulación de estrategias requiere buenas destrezas conceptuales, integradoras y analíticas, la ejecución necesita habilidades específicas en cuanto a motivación y administración del personal. La formulación de estrategias ocurre primordialmente en los niveles gerenciales de una organización, mientras que la ejecución de estas incluye todos los niveles jerárquicos.

En lo que respecta a la ejecución, David (2008), incluye acciones tales como cambios en los territorios de ventas, adición de nuevos departamentos, cierre de instalaciones, contratación de nuevos empleados, cambios en la estrategia de precios, desarrollo de nuevas prestaciones del personal, fijación de procedimientos de control de costos, cambios en las estrategias publicitarias, construcción de instalaciones, adiestramientos de nuevos empleados y transferencias de gerentes entre divisiones.

Por su parte, Betancourt (2002), plantea que la ejecución de estrategias afecta todas las esferas de una organización, causando impacto en todas las áreas funcionales de la misma. De tal forma, la 
ejecución de estrategias causa impacto directamente en las vidas de los gerentes de mercadeo, finanzas, producción, investigación y desarrollo, planta, división, ventas, productos, proyectos de personal, y de los demás empleados, aunque ellos no hayan participado en el proceso de formulación de estrategias.

Al respecto de lo expuesto, Sallenave (2009) refiere que los gerentes que implantan el proceso de gerencia estratégica en áreas operacionales lo hacen por varias razones, entre las cuales menciona las siguientes: creencia de que ésta aumenta la eficiencia operacional; y estimula sentimientos de autorrealización y satisfacción, ya que a medida que los directivos y planificadores trabajan juntos para formular planes de estrategia e implementación, adquieren confianza en su capacidad para interpretar y hacer frente a la situación, esto tiende a ser muy grato y satisfactorio, facilita la adaptación al cambio.

Desde esta óptica, a juicio del investigador, la gestión estratégica de operaciones es un proceso mediante el cual pueden coordinarse los esfuerzos de divisiones, funciones e individuos para el logro de metas y objetivos organizacionales, siendo necesaria su implementación cuando se producen importantes reorganizaciones o cambios en la gerencia de la empresa, contribuyendo a formar el nuevo equipo de gerencia en los aspectos de oportunidades y restricciones internas y externas que deberán afrontar los miembros del equipo.

\section{Estrategias de operaciones}

Para Caicedo (2009), la estrategia de operaciones se basa en un plan a largo plazo que busca mejorar la competitividad. Inicia con un análisis del entorno, mercado y competidores, así como de un estudio de los recursos internos disponibles, para fijar objetivos y hoja de ruta.

Explica este autor que, el objetivo final de la estrategia de operaciones es encontrar una ventaja competitiva que diferencie claramente a la compañía de sus competidores. Se trata de, el valor agregado del producto o servicio ofrecidos justifique un precio superior en el producto final que el cliente esté no sólo dispuesto a pagarlo, sino satisfecho de hacerlo. Esta ventaja debe ser sostenible en el tiempo y de difícil imitación.

Coincidiendo con Nahmias (2007), quien menciona que las estrategias de operaciones es la manera en que la empresa utiliza sus recursos para lograr sus metas competitivas. Dentro de este marco de referencia, Wheelen y Hunger (2007) definen la estrategia de operaciones como directrices que ayudan a elegir cursos de acción adecuados para alcanzar metas que la organización se ha fijado.

Según los autores, estas estrategias deben ser conducentes a los objetivos generales organizacionales, y tienen la mayor importancia porque acarrean consecuencias para toda la organización, siendo el origen de las políticas de empresa y de todo el resto de la planificación. Involucra decisiones relativas al diseño del proceso y a la infraestructura que se necesita para apoyarlos.

Ahora bien, Nahmias (2007) establece que las estrategias de operaciones pueden ser de muy diversa índole y pueden hacer hincapié en aspectos competitivos diferentes. Visto así, para el investigador, las estrategias de operaciones son guías que ayudan a elegir cursos de acción viables para alcanzar las metas operacionales de la empresa.

\section{METODO}

El presente estudio se plasmó como una investigación descriptiva, con diseño no experimental, transeccional, y de campo. La población quedó compuesta por las cuatro (4) empresas mixtas de la industria petrolera venezolana en la Costa Sur del Lago Trujillo, activas al momento de realizar el estudio, siendo éstas: Petroquiriquire S.A., Petrolera SINO-Venezolana S.A., Lagopetrol S.A., y Patrozamora S.A.

Las unidades informantes quedaron representadas por los gerentes generales, gerentes 
de operaciones de producción y gerentes de operaciones de mantenimiento, estimándose un total de 12 sujetos informantes, a quienes se les aplico un cuestionario conformado por 57 ítems con 5 alternativas de respuestas: totalmente de acuerdo $=5 ;$ de acuerdo $=4 ;$ ni de acuerdo $/$ ni en desacuerdo $=3$; desacuerdo $=2$; totalmente en desacuerdo $=1$. Se contó con el juicio de 5 expertos en el área de gestión estratégica de operaciones, así como de metodología y para la confiabilidad se utilizó el método estadístico Alpha de Cronbach, obteniendo un coeficiente de 0,9321 , demostrando alta confiabilidad del instrumento.

RESULTADOS

El análisis de los resultados se realizó con base en la estadística descriptiva, a través de la distribución de frecuencias relativas y la medida de tendencia central media aritmética. Estos han sido estructurados en tablas contentivas de toda la información recogida sobre la variable, clasificada por dimensión e indicador.

Tabla 1. Dimensión: proceso de operaciones.

INDICADOR: DECISIÓN DEL PROCESO

\begin{tabular}{|c|c|c|c|c|c|}
\hline No. & ÍTEMS & $\mathrm{TA}+\mathrm{DA}$ & TENDENCIA & MEDIA & CATEGORÍA \\
\hline 1 & $\begin{array}{l}\text { Analizan la interacción de sus procesos, } \\
\text { asegurándose de que la secuencia e interacción } \\
\text { de los mismos se diseñen con el fin de lograr los } \\
\text { resultados deseados en los planes empresariales. }\end{array}$ & 100 & Positiva & 4,83 & Muy alto cumplimiento \\
\hline 2 & $\begin{array}{l}\text { Al momento de diseñar los procesos se visualiza el } \\
\text { alcance de ventajas competitivas. }\end{array}$ & 91,67 & Positiva & 4,42 & Muy alto cumplimiento \\
\hline \multirow[t]{2}{*}{3} & Examinan cada paso de sus procesos buscando & 100 & Positiva & 4,50 & Muy alto cumplimiento \\
\hline & Promedio Indicador & 97,22 & Positiva & 4,58 & Muy alto cumplimiento \\
\hline \multicolumn{6}{|c|}{ INDICADOR: DISEÑO DEL PROCESO } \\
\hline No. & ÍTEMS & $\mathrm{TA}+\mathrm{DA}$ & TENDENCIA & MEDIA & CATEGORÍA \\
\hline 4 & $\begin{array}{l}\text { Alos procesos son diseñados para obtener } \\
\text { resultados de bajo costo. }\end{array}$ & 83,33 & Positiva & 4,58 & Muy alto cumplimiento \\
\hline 5 & $\begin{array}{l}\text { Invierten en rediseñar sus procesos para optimizar } \\
\text { los recursos. }\end{array}$ & 100 & Positiva & 4,67 & Muy alto cumplimiento \\
\hline \multirow[t]{2}{*}{6} & Cuentan con normas/procedimientos por escrito & 100 & Positiva & 4,75 & Muy alto cumplimiento \\
\hline & Promedio Indicador & 94,44 & Positiva & 4,67 & Muy alto cumplimiento \\
\hline
\end{tabular}




\section{INDICADOR: CONTROL DE PROCESO}

\begin{tabular}{|c|c|c|c|c|c|}
\hline No. & ÍTEMS & $\mathbf{T A}+\mathbf{D A}$ & TENDENCIA & MEDIA & CATEGORÍA \\
\hline 7 & $\begin{array}{l}\text { Se miden los esultados de los procedimientos de } \\
\text { la calidad. }\end{array}$ & 83,33 & Positiva & 4,83 & Muy alto cumplimiento \\
\hline 8 & $\begin{array}{l}\text { Evalúan el flujo de las diferentes áreas de } \\
\text { producción a través de indicadores. }\end{array}$ & 100 & Positiva & 4,92 & Muy alto cumplimiento \\
\hline \multirow[t]{2}{*}{9} & $\begin{array}{l}\text { Los procesos comprenden sistemas flexibles de } \\
\text { producción con fácil manejo. }\end{array}$ & 100 & Positiva & 4,75 & Muy alto cumplimiento \\
\hline & Promedio Indicador & 94,44 & Positiva & 4,67 & Muy alto cumplimiento \\
\hline \multicolumn{6}{|c|}{ INDICADOR: MEJORAS DEL PROCESO } \\
\hline No. & ITTEMS & $\mathrm{TA}+\mathrm{DA}$ & TENDENCIA & MEDIA & CATEGORÍA \\
\hline 10 & $\begin{array}{l}\text { Verifican la optimización de los recursos durante } \\
\text { la actividad de producción. }\end{array}$ & 100 & Positiva & 4,83 & Muy alto cumplimiento \\
\hline 11 & $\begin{array}{l}\text { Llevan a cabo auditorías internas para hacer } \\
\text { seguimiento a sus procesos. }\end{array}$ & 100 & Positiva & 4,92 & Muy alto cumplimiento \\
\hline \multirow[t]{2}{*}{12} & $\begin{array}{l}\text { Fijan objetivos de satisfacción del cliente, para } \\
\text { conducir la ejecución de los procesos. }\end{array}$ & 58,33 & Neutral & 3,83 & Alto cumplimiento \\
\hline & Promedio Indicador & 86,11 & Positiva & 4,53 & Muy alto cumplimiento \\
\hline \multicolumn{6}{|c|}{ INDICADOR: SELECCIÓN DEL PROCESO } \\
\hline No. & ÍTEMS & $\mathrm{TA}+\mathrm{DA}$ & TENDENCIA & MEDIA & CATEGORÍA \\
\hline 13 & $\begin{array}{l}\text { Realizan estudios de tiempos en las líneas de } \\
\text { producción. }\end{array}$ & 50.00 & Neutral & 3,67 & Alto cumplimiento \\
\hline 14 & $\begin{array}{l}\text { Eliminan los procesos que no añaden valor al } \\
\text { producto. }\end{array}$ & 100 & Positiva & 4,75 & Muy alto cumplimiento \\
\hline \multirow[t]{3}{*}{15} & $\begin{array}{l}\text { Cumplen con las fechas de corrección en las } \\
\text { desviaciones encontradas durante la auditoría } \\
\text { interna. }\end{array}$ & 66.67 & Neutral & 3,83 & Alto cumplimiento \\
\hline & Promedio Indicador & 72,22 & Positiva & 4,08 & Alto cumplimiento \\
\hline & PROMEDIO DIMENSIÓN & 89,44 & Positiva & 4,53 & Muy alto cumplimiento \\
\hline
\end{tabular}

Por consiguiente, al hacer referencia al primer objetivo específico, orientado a describir el proceso de operaciones presente en las empresas mixtas de la industria petrolera venezolana en la Costa Sur del Lago-Trujillo, se evidencia en la tabla 1, el 89,44\% en promedio de los encuestados respondieron a los ítems involucrados en las alternativas totalmente de acuerdo y de acuerdo, indicando una tendencia positiva en las respuestas emitidas. Al mismo tiempo, se observa una media para la dimensión de 4,53 implicando muy alto cumplimiento del proceso de operaciones, como parte de la gestión estratégica de operaciones que realizan las empresas analizadas. 
En específico, todos los indicadores utilizados ostentan muy alto cumplimiento, a excepción de la selección del proceso calificada con alto cumplimiento, observándose esto en las medias alcanzadas por dichos indicadores. Al mismo tiempo se observan tendencias positivas para todos los indicadores, donde todos los promedios de las respuestas emitidas superan el $70 \%$ en la suma de las opciones positivas (TA + DA).

Este muy alto cumplimiento de la dimensión proceso de operaciones, valida lo expuesto por Rodríguez (2008), para quien el proceso de operaciones, tiene como finalidad determinar criterios y métodos para asegurar que las actividades que comprende dicho proceso se realicen de manera eficaz, al igual que el control del mismo.

De igual manera validan al investigador cuando afirma que, en virtud de obtener la satisfacción total del cliente y lograr altos niveles de rendimiento financiero, es necesario analizar los procesos operacionales de la organización, con la finalidad de detectar las áreas críticas y poder aplicar los correctivos necesarios de forma oportuna.
Al detalle se aprecia que todos los indicadores, considerados en esta investigación: tipos, planificación y flexibilidad de la capacidad, arribaron a la categoría de muy alto cumplimiento con medias que así lo ratifican. No obstante, en cada indicador se observan actividades ubicadas en la categoría alto cumplimiento, lo cual es indicativo de oportunidades de mejora en estos aspectos. Además, se acota que, todos los indicadores muestran tendencia positiva de las respuestas obtenidas.

Estos resultados logran validar a Krajewski y Ritzman (2008), para quienes la capacidad del proceso de operaciones es la tasa de producción máxima de una instalación, que puede ser una estación de trabajo u organización entera. El gerente de operaciones debe suministrar la capacidad necesaria para satisfacer la demanda actual y futura; de otra forma la organización perdería oportunidades de crecimiento y de ganar beneficios.

Tabla 2. Dimensión: capacidad del proceso de operaciones.

\section{INDICADOR: TIPOS DE CAPACIDAD}

\begin{tabular}{clcccc}
\hline No. & \multicolumn{1}{c}{ ÍTEMS } & TA+DA & TENDENCIA & MEDIA & CATEGORÍA \\
16 & $\begin{array}{l}\text { El volumen de producción por período permite } \\
\text { cubrir los costos mínimos. }\end{array}$ & 58,33 & Neutral & 3,58 & Alto cumplimiento \\
17 & $\begin{array}{l}\text { Basan las operaciones a la máxima eficiencia del } \\
\text { 100\% del tiempo, es decir, a la capacidad para la } \\
\text { que se diseño el sistema. }\end{array}$ & 100 & Positiva & 4,58 & Muy alto cumplimiento \\
18 & $\begin{array}{l}\text { Planifican las operaciones con base a la capacidad } \\
\text { de planta utilizada, ajustándose a la demanda } \\
\text { actual. }\end{array}$ & 83,33 & Positiva & 4,25 & Muy alto cumplimiento \\
19 & $\begin{array}{l}\text { Al planificar su producción se ajustan a la } \\
\text { utilización de los equipos según los registros de } \\
\text { las demandas pasadas. }\end{array}$ & 83,33 & Positiva & 4,33 & Muy alto cumplimiento \\
20 & $\begin{array}{l}\text { La capacidad de operaciones es calculada según } \\
\text { mediciones basadas en la salida del producto. }\end{array}$ & 83,33 & Positiva & 4,50 & Muy alto cumplimiento \\
\hline Promedio Indicador & $\mathbf{8 1 , 6 7}$ & Positiva & $\mathbf{4 , 2 5}$ & Muy alto cumplimiento \\
\hline
\end{tabular}




\begin{tabular}{|c|c|c|c|c|c|}
\hline \multicolumn{6}{|c|}{ INDICADOR: PLANIFICACIÓN DE LA CAPACIDAD } \\
\hline No. & ÍTEMS & $\mathrm{TA}+\mathrm{DA}$ & TENDENCIA & MEDIA & CATEGORÍA \\
\hline 21 & $\begin{array}{l}\text { Consideran la planificación } \\
\text { semanal je producción } \\
\text { involucrados. }\end{array}$ & 100 & Positiva & 4,75 & Muy alto cumplimiento \\
\hline 22 & $\begin{array}{l}\text { Definen con anterioridad cuando se debe } \\
\text { subcontratar personal en las áreas de operaciones. }\end{array}$ & 50 & Neutral & 3,42 & Alto cumplimiento \\
\hline 23 & $\begin{array}{l}\text { Consideran la planificación de producción a } \\
\text { mediano plano junto con los departamentos } \\
\text { involucrados. }\end{array}$ & 100 & Positiva & 4,42 & Alto cumplimiento \\
\hline 24 & $\begin{array}{l}\text { Consideran la planificación de producción a largo } \\
\text { plazo junto con los departamentos involucrados. }\end{array}$ & 91,67 & Positiva & 4,33 & Muy alto cumplimiento \\
\hline 25 & $\begin{array}{l}\text { Proyectan la disponibilidad de equipos durante } \\
\text { todo el horizonte de planificación. }\end{array}$ & 83,33 & Positiva & 4,25 & Muy alto cumplimiento \\
\hline & Promedio Indicador & 85,00 & Positiva & 4,23 & Muy alto cumplimiento \\
\hline \multicolumn{6}{|c|}{ INDICADOR: FLEXIBILIDAD DE LA CAPACIDAD } \\
\hline No. & ÍTEMS & $\mathrm{TA}+\mathrm{DA}$ & TENDENCIA & MEDIA & CATEGORÍA \\
\hline 26 & $\begin{array}{l}\text { Las áreas de producción presentan un tamaño que } \\
\text { permite que las instalaciones puedan cumplir con } \\
\text { el plan de mercadeo. }\end{array}$ & 75 & Positiva & & Alto cumplimiento \\
\hline 27 & $\begin{array}{l}\text { Tienen conocimiento de lo que se debe invertir en } \\
\text { nuevos equipos para incrementar la producción. }\end{array}$ & 100 & Positiva & & Muy alto cumplimiento \\
\hline 28 & $\begin{array}{l}\text { Cuentan con personal capacitado para realizar sus } \\
\text { operaciones. }\end{array}$ & 100 & Positiva & & Muy alto cumplimiento \\
\hline 29 & $\begin{array}{l}\text { Los gerentes tienen un liderazgo que obtiene lo } \\
\text { mejor del desempeño de sus empleados. }\end{array}$ & 100 & Positiva & & Muy alto cumplimiento \\
\hline \multirow[t]{3}{*}{30} & $\begin{array}{l}\text { El trabajador se apoya en programas de formación } \\
\text { para cambiar de un tipo de tarea a otro. }\end{array}$ & 66,67 & Neutral & & Alto cumplimiento \\
\hline & Promedio Indicador & 88,33 & Positiva & 4,50 & Muy alto cumplimiento \\
\hline & PROMEDIO DIMENSIÓN & 85,00 & Positiva & 4,33 & Muy alto cumplimiento \\
\hline
\end{tabular}

Dado estos resultados, el investigador asume que en las empresas mixtas de la industria petrolera venezolana en la Costa Sur del Lago-Trujillo se trabaja acorde a la capacidad de operaciones con la que cuentan, para satisfacer los niveles de su demanda actual aprovechando las oportunidades que esta capacidad le brinda.

En la tabla 3, se presenta el resumen para esta dimensión, reflejando, de acuerdo a los encuestados, una concentración de respuestas afirmativas (TA + DA) del $88,89 \%$, indicando tendencia positiva de las mismas. También se evidencia una media para la dimensión de 4,40, indicando que, en las empresas mixtas bajo estudio, se da muy alto cumplimiento al proceso de gestión estratégica de operaciones.

Estos resultados logran muy alta congruencia con lo expuesto por Sallenave (2009), para quien el proceso de gestión estratégica de operaciones puede resumirse en una secuencia de pasos, los cuales pueden ayudar a preparar el análisis de caso de política empresarial. Estos pasos son agrupados en tres (3) etapas, siendo éstas: formulación, ejecución y evaluación estratégica. 
Tabla 3. Dimensión: proceso de gestión estratégica de operaciones.

\begin{tabular}{|c|c|c|c|c|c|}
\hline \multicolumn{6}{|c|}{ INDICADOR: FORMULACIÓN ESTRATÉGICA } \\
\hline No. & ÍTEMS & $\mathrm{TA}+\mathrm{DA}$ & TENDENCIA & MEDIA & CATEGORÍA \\
\hline 31 & $\begin{array}{l}\text { Realizan actividades de investigación con el } \\
\text { objeto de identificar tanto las debilidades como } \\
\text { las fortalezas claves relacionadas al área de } \\
\text { operaciones }\end{array}$ & 91,67 & Positiva & 4,33 & Muy alto cumplimiento \\
\hline 32 & $\begin{array}{l}\text { Realizan actividades de investigación con el objeto } \\
\text { de identificar tanto las oportunidades como las } \\
\text { amenazas claves externas de la organización }\end{array}$ & 83,33 & Positiva & 4,17 & Alto cumplimiento \\
\hline \multirow[t]{2}{*}{33} & $\begin{array}{l}\text { Formulan estrategias de operaciones considerando } \\
\text { los factores tanto internos como externos que } \\
\text { inciden en la prestación del servicio }\end{array}$ & 100 & Positiva & 4,58 & Muy alto cumplimiento \\
\hline & Promedio Indicador & 91,67 & Positiva & 4,36 & Muy alto cumplimiento \\
\hline \multicolumn{6}{|c|}{ INDICADOR: EJECUCIÓN ESTRATÉGICA } \\
\hline No. & ÍTEMS & $\mathrm{TA}+\mathrm{DA}$ & TENDENCIA & MEDIA & CATEGORÍA \\
\hline 34 & $\begin{array}{l}\text { Las gerencias de operaciones utilizan indicadores } \\
\text { que permitan medir el desempeño. }\end{array}$ & 100 & Positiva & 4,75 & Muy alto cumplimiento \\
\hline 35 & $\begin{array}{l}\text { Las políticas formuladas estimulan al talento } \\
\text { humano a trabajar para la consecución de las } \\
\text { metas fijadas. }\end{array}$ & 91,67 & Positiva & 4,42 & Muy alto cumplimiento \\
\hline \multirow[t]{2}{*}{36} & $\begin{array}{l}\text { Los recursos organizacionales son asignados de } \\
\text { acuerdo con las prioridades establecidas en las } \\
\text { metas. }\end{array}$ & 66,67 & Neutral & 3,83 & Alto cumplimiento \\
\hline & Promedio Indicador & 86,11 & Positiva & 4,33 & Muy alto cumplimiento \\
\hline \multicolumn{6}{|c|}{ INDICADOR: EVALUACIÓN ESTRATÉGICA } \\
\hline No. & ÍTEMS & $\mathrm{TA}+\mathrm{DA}$ & TENDENCIA & MEDIA & CATEGORÍA \\
\hline 37 & $\begin{array}{l}\text { Las gerencias de operaciones utilizan indicadores } \\
\text { que permitan medir el desempeño. }\end{array}$ & 100 & Positiva & & Muy alto cumplimiento \\
\hline 38 & $\begin{array}{l}\text { Implementan medidas correctivas oportunamente } \\
\text { requeridas para mejorar la posición estratégica de } \\
\text { la empresa. }\end{array}$ & 91,67 & Positiva & & Muy alto cumplimiento \\
\hline \multirow[t]{3}{*}{39} & $\begin{array}{l}\text { Disponen de estrategias opcionales a implementar } \\
\text { cuando los factores cambian de forma significativa. }\end{array}$ & 75 & Positiva & & Alto cumplimiento \\
\hline & Promedio Indicador & 88,89 & Positiva & 4,50 & Muy alto cumplimiento \\
\hline & PROMEDIO DIMENSIÓN & 88,89 & Positiva & 4,40 & Muy alto cumplimiento \\
\hline
\end{tabular}

Lo mostrado permite al investigador afirmar que, en la gestión estratégica de operaciones de las empresas mixtas analizadas, se ha logrado establecer muy alto nivel de reconocimiento de la importancia que debe dársele al proceso de gestión estratégica de operaciones. De tal forma, para estas empresas, la gestión estratégica de operaciones es un proceso mediante el cual pueden coordinarse los esfuerzos de divisiones, funciones e individuos para el logro de metas y objetivos organizacionales. Comprendiendo que la gestión estratégica es un proceso gerencial debidamente planificado, en el cual se toman un conjunto de decisiones interactivas y superpuestas conducentes al desarrollo de una 
estrategia eficaz para la empresa

Prosiguiendo el análisis, se presentan ahora los resultados obtenidos para la cuarta y última dimensión dada a la variable, denominada estrategias de operaciones, y que se deriva del cuarto objetivo específico, en el cual se desea determinar las estrategias de operaciones presentes en las empresas mixtas de la industria petrolera en la Costa Sur del Lago-Trujillo.

Al respecto, en la tabla 4 se muestran los resultados del trabajo de campo para esta dimensión. Como puede apreciarse, esta dimensión ostenta alto cumplimiento con una media de 3,81 , y tendencia neutral de las respuestas obtenidas, dado que el promedio de respuestas en las opciones totalmente de acuerdo y de acuerdo se ubicó en $59,72 \%$.

Adicionalmente, puede observarse muy alto cumplimiento solo para el indicador calidad $(4,58)$, mientras el resto de los indicadores fueron categorizados con alto cumplimiento: costos $(3,94)$, velocidad y confiabilidad en la entrega $(3,50)$, capacidad para afrontar cambios de la demanda $(3,81)$, flexibilidad y velocidad para la introducción de nuevos productos o servicios $(3,47)$ y criterios del servicio $(3,56)$. También se evidencia tendencia positiva de las respuestas emitidas solo para el indicador calidad, el resto de los indicadores evidenciaron tendencias neutrales.

La situación presentada, donde las estrategias de operaciones muestran alto cumplimiento, coinciden en alto grado con lo postulado por Nahmias (2007) cuando refiere que, toda estrategia de operaciones debe centrar su atención en la satisfacción del cliente lo cual implicaría el desarrollo de estrategias dirigidas a: calidad del producto; rapidez en la entrega; eficiencia en la prestación de los servicios; control de los costos; capacidad de afrontar cambios en la demanda; y flexibilidad y velocidad para la introducción de nuevos servicios.

Por lo evidenciado, para el investigador, las empresas mixtas bajo estudio han logrado una percepción clara de la importancia adjudicada al uso de estrategias de operaciones para lograr efectividad de su gestión estratégica de operaciones, comprendiendo que estas estrategias son guías que les ayudan a elegir cursos de acción viables para alcanzar las metas operacionales de la empresa. No obstante, al posicionarse en alto y no muy alto cumplimiento deja ver oportunidades de mejora en los aspectos medidos.

Tabla 4. Dimensión: estrategias de operaciones.

INDICADOR: COSTOS

\begin{tabular}{cccccc}
\hline No. & \multicolumn{1}{c}{ ÍTEMS } & TA+DA & TENDENCIA & MEDIA & CATEGORÍA \\
40 & $\begin{array}{l}\text { Fundamentan su estrategia de operaciones en } \\
\text { la reducción de los costos involucrados en la } \\
\text { prestación del servicio. }\end{array}$ & 91,67 & Positiva & 4,67 & Muy alto cumplimiento \\
41 & $\begin{array}{l}\text { Fundamentan su estrategia de operaciones } \\
\text { en el liderazgo en costos. }\end{array}$ & 100 & Positiva & 4,67 & Alto cumplimiento \\
42 & $\begin{array}{l}\text { Los servicios de operaciones son ofrecidos } \\
\text { a costo más bajos en comparación a las } \\
\text { empresas competidoras. }\end{array}$ & Negativa & 2,50 & Mediano cumplimiento \\
\hline & Promedio Indicador & $\mathbf{6 6 , 6 7}$ & Neutral & $\mathbf{3 , 9 4}$ & Muy alto cumplimiento \\
\hline
\end{tabular}




\section{INDICADOR: CALIDAD}

\begin{tabular}{|c|c|c|c|c|c|}
\hline No. & ÍTEMS & $\mathrm{TA}+\mathrm{DA}$ & TENDENCIA & MEDIA & CATEGORÍA \\
\hline 43 & $\begin{array}{l}\text { Fundamentos su estrategia de operaciones en el } \\
\text { mejoramiento continuo de la calidad del servicio. }\end{array}$ & 100 & Positiva & 4,75 & Muy alto cumplimiento \\
\hline 44 & $\begin{array}{l}\text { Se esfuerzan en prestar el servicio de acuerdo a los } \\
\text { parámetros de calidad internacionales. }\end{array}$ & 83,33 & Positiva & 4,67 & Muy alto cumplimiento \\
\hline \multirow[t]{3}{*}{45} & Se esfuerza en cumplir con los requerimientos & 75 & Positiva & 4,33 & Mediano cumplimiento \\
\hline & Promedio Indicador & 86,11 & Positiva & 4,58 & Muy alto cumplimiento \\
\hline & \multicolumn{5}{|c|}{ INDICADOR: VELOCIDAD Y CONFIABILIDAD EN LA ENTREGA } \\
\hline No. & ÍTEMS & $\mathrm{TA}+\mathrm{DA}$ & TENDENCIA & MEDIA & CATEGORÍA \\
\hline 46 & $\begin{array}{l}\text { Fundamentan su estrategia de operaciones en la } \\
\text { reducción del tiempo de entrega de los resultados. }\end{array}$ & 100 & Positiva & 4,50 & Muy alto cumplimiento \\
\hline 47 & $\begin{array}{l}\text { Cumplen con los lapsos de entrega de los resultados } \\
\text { acordados con los clientes. }\end{array}$ & 33,33 & Negativa & 3,08 & Alto cumplimiento \\
\hline \multirow[t]{3}{*}{48} & $\begin{array}{l}\text { El tiempo de entrega de los resultados es menor al } \\
\text { ofrecido por las empresas competidoras. }\end{array}$ & 25 & Negativa & 2,92 & Mediano cumplimiento \\
\hline & Promedio Indicador & 52,78 & Neutral & 3,50 & Alto cumplimiento \\
\hline & \multicolumn{5}{|c|}{ INDICADOR: CAPACIDAD PARA AFRONTAR CAMBIOS DE LA DEMANDA } \\
\hline No. & ÍTEMS & $\mathrm{TA}+\mathrm{DA}$ & TENDENCIA & MEDIA & CATEGORÍA \\
\hline 49 & $\begin{array}{l}\text { Analizan los cambios que se producen en el entorno } \\
\text { de la actividad petrolera nacional e internacional. }\end{array}$ & 75 & Positiva & 4,33 & Muy alto cumplimiento \\
\hline 50 & $\begin{array}{l}\text { Adaptan los servicios que prestan a las necesidades } \\
\text { de sus clientes. }\end{array}$ & 50,00 & Neutral & 3,67 & Alto cumplimiento \\
\hline \multirow[t]{3}{*}{51} & $\begin{array}{l}\text { Realizan estudios que les permita anticiparse a los } \\
\text { cambios en los servicios demandados por los clientes. }\end{array}$ & 41,67 & Neutral & 3,42 & Alto cumplimiento \\
\hline & Promedio Indicador & 55,56 & Neutral & 3,81 & Alto cumplimiento \\
\hline & \multicolumn{5}{|c|}{$\begin{array}{l}\text { INDICADOR: FLEXIBILIDAD Y VELOCIDAD PARA LA INTRODUCCIÓN } \\
\text { DE NUEVOS PRODUCTOS O SERVICIOS }\end{array}$} \\
\hline 52 & $\begin{array}{l}\text { Fundamentan su estrategia de operaciones en el } \\
\text { ofrecimiento de diversidad de servicios relacionados } \\
\text { con el servicio prestado }\end{array}$ & 58,33 & Neutral & 3,75 & Alto cumplimiento \\
\hline 53 & $\begin{array}{l}\text { Invierten en actividades de investigación y desarrollo } \\
\text { con la finalidad de desarrollar nuevos servicios. }\end{array}$ & 33,33 & Negativa & 3,25 & Alto cumplimiento \\
\hline \multirow[t]{2}{*}{54} & $\begin{array}{l}\text { Dedican tiempo a la transformación de sus procesos } \\
\text { con la finalidad de ofrecer nuevos servicios. }\end{array}$ & 41,67 & Neutral & 3,42 & Alto cumplimiento \\
\hline & Promedio Indicador & 44,44 & Neutral & 3,47 & Alto cumplimiento \\
\hline
\end{tabular}




\section{INDICADOR: CRITERIOS DEL SERVICIO}

\begin{tabular}{cccccc}
\hline No. & \multicolumn{1}{c}{ İTEMS } & TA+DA & TENDENCIA & MEDIA & CATEGORÍA \\
55 & $\begin{array}{l}\text { Proporcionan ayuda técnica especializada a sus } \\
\text { clientes con la finalidad de explicar los resultados } \\
\text { obtenidos. }\end{array}$ & 50 & Neutral & 3,58 & Alto cumplimiento \\
56 & $\begin{array}{l}\text { Están en capacidad de trabajar coordinadamente } \\
\text { con otras empresas durante la prestación del } \\
\text { servicio. }\end{array}$ & 83,33 & Positiva & 4,33 & Muy alto cumplimiento \\
57 & $\begin{array}{l}\text { Tienen la capacidad de modificar los servicios } \\
\text { que prestan en la actualidad para que alcancen } \\
\text { nuevos niveles de desempeño de acuerdo a los } \\
\text { requerimientos de los clientes. }\end{array}$ & Negativa & 25,75 & Mediano cumplimiento \\
Promedio Indicador & Neutral & $\mathbf{4 , 5 0}$ & Alto cumplimiento \\
\hline & Neutral & $\mathbf{4 , 3 3}$ & Alto cumplimiento \\
\hline
\end{tabular}

Finalmente, se muestran en la tabla 5, los resultados obtenidos para la variable gestión estratégica de operaciones. Como puede observarse, la gestión estratégica de operaciones, en las empresas mixtas mencionadas, fue catalogada en la categoría de excelente gestión con una media de 4,27; y tendencia positiva de las respuestas emitidas por los encuestados, al obtener una concentración en las respuestas afirmativas (TA+DA) de 80,76\%.
Al detalle, se visualiza muy alto cumplimiento para las dimensiones proceso de operaciones, capacidad del proceso de operaciones, proceso de gestión estratégica de operaciones, con tendencias positivas en las respuestas dadas; mientras, catalogaron con alto cumplimiento las estrategias de operaciones, teniendo tendencia neutral en las respuestas emitidas.

Tabla 5. Variable: gestión estratégica de operaciones.

\begin{tabular}{lcccc}
\hline \multicolumn{1}{c}{ DIMENSIÓN } & TA + DA & TENDENCIA & MEDIA & CATEGARÍA \\
\hline Proceso de Operaciones & 89,44 & Positiva & 4,53 & Muy alto cumplimiento \\
Capacidad del Proceso de Operaciones & 85,00 & Positiva & 4,33 & Muy alto cumplimiento \\
Proceso de Gestión Estratégica de Operaciones & 88,89 & Positiva & 4,40 & Muy alto cumplimiento \\
& & & & Alto cumplimiento \\
Estrategias de Operaciones & 59,72 & Positiva & 3,81 & Excelente \\
\hline
\end{tabular}


Esta calificación de excelente gestión permite validar lo postulado por Muñoz (2009), para quien la gestión estratégica de operaciones es la guía, conducción y control de los esfuerzos de un grupo de individuos hacia un objetivo común, es el proceso que permite optimizar la función de producción, aumentar la calidad, mejorar la satisfacción de los clientes y disminuir los costos, fundamentado en la planificación para determinar qué se va a hacer, la toma de decisiones que incluyen el esclarecimiento de objetivos, establecimiento de políticas, fijación de programas-campañas, determinación de métodos o procedimientos específicos y fijación de previsiones.

En virtud de lo alcanzado, se visualiza concordancia con la posición del investigador cuando afirma que, la gestión estratégica de operaciones proporciona una visión global en cuanto al tipo de empresa que la organización desea ser, guiando el conjunto de decisiones de planificación, organización, dirección y control que generen acciones presentes y futuras en las áreas de producción de bienes o servicios que comprende su diseño, procesos de trabajo, generación, calidad, así como actividades de localización, abastecimiento e inventario y mantenimiento, con la finalidad de generar ventajas competitivas sostenibles.

\section{CONCLUSIONES}

En relación con el proceso de operaciones presente, se concluye que estas empresas poseen muy alto cumplimiento, de manera global, de este proceso en la gestión estratégica de operaciones que realizan. Debe mencionarse que, aun dada esta categoría de muy alto cumplimiento, se detectó la existencia de ciertos aspectos establecidos como determinantes para medir esta dimensión, los cuales deben ser reforzados dado que ofrecen oportunidades de mejora.

Específicamente en cuanto a las actividades de mejoras del proceso y selección del proceso, dado que, según los encuestados, poseen aspectos involucrados a estas actividades evaluados en las categorías de alto cumplimiento, motivo por el cual deben revisarlos, en virtud de la importancia que tienen estos aspectos en la efectividad de la gestión estratégica de operaciones, y de ello dependerá el desarrollo de dicha gestión a través del logro de sus objetivos.

En lo que concierne a la capacidad del proceso de operaciones, se concluye que estas empresas evidenciaron muy alto cumplimiento de todas las actividades involucradas a esta capacidad, que se basa en los tipos de capacidad, planificación de la capacidad y flexibilidad de la capacidad.

Es necesario destacar que, si bien se obtuvo muy alto cumplimiento de la capacidad del proceso de operaciones en la gestión analizada, se detectaron oportunidades de mejora en las actividades medidas para los tipos de capacidad, planificación de la capacidad y flexibilidad de la capacidad, lo cual permite al investigador establecer acciones estratégicas referidas a este aspecto en las recomendaciones del estudio.

Ahora bien, en relación con el proceso de gestión estratégica de operaciones, se concluye que en estas empresas el proceso de gestión estratégica de operaciones posee muy alto cumplimiento; no obstante, se detectaron oportunidades de mejora en sus tres (3) indicadores, a saber: formulación, ejecución y evaluación estratégica, los cuales evidenciaron actividades medidas a través de sus ítems catalogadas con alto cumplimiento, y no así muy alto.

En cuanto a las estrategias de operaciones, se evidencio que estas estrategias poseen alto cumplimiento en la gestión estratégica que desarrollan estas empresas. Cabe resaltar que, para este estudio fueron consideradas seis (6) estrategias de operaciones, de las cuales solo una (calidad) logró posicionarse en muy alto cumplimiento, en conjunto y al detalle de todas las actividades medidas; el resto de las estrategias consideradas se posicionaron en alto cumplimiento, reflejando 
actividades con alto y mediano cumplimiento, siendo susceptibles de mejora y serán la base de las recomendaciones para este aspecto.

Atendiendo las consideraciones anteriores, con respecto a la variable gestión estratégica de operaciones, se concluye existe una excelente gestión estratégica de operaciones. Así las cosas, se mostró muy alto cumplimiento del proceso de operaciones, la capacidad del proceso de operaciones y el proceso de gestión estratégica de operaciones; y alto cumplimiento de las estrategias de operaciones.

Se debe resaltar, que aun cuando los resultados de excelente gestión estratégica de operaciones se constituyen en una ventaja competitiva para el sector en estudio, se dan algunas actividades, relacionadas a los ítems utilizados para medir los indicadores, mostrando oportunidades de mejora dado se ubican en la categoría de alto y mediano cumplimiento.

\section{REFERENCIAS}

Arnoletto, E. (2010). Técnicas polito-lógicas para la gestión de productos sociales. En: $\quad$ http://www.academia.edu/1308540/ T\%C3\%A9cnicas_politol\%C3\%B3gicas_para_ la_gesti\%C3\%B3n_de_proyectos_sociales

Betancourt, J. (2002). Gestión Estratégica: Navegando hacia el cuarto paradigma. Aspectos conceptuales. 3era. Edición. Editorial T.G. Red 2000 Ediciones. Venezuela

Caicedo, A. (2009). Estrategia corporativa y de operaciones. Disponible en: http:// www.slideshare.net/krmen01/estrategiacorportativa-y-de-operaciones

Chase, R.; Aquilano, N. Y Jacobs, R. (2009). Administración de operaciones "producción y cadena de suministros". (12० ed.). Editorial McGraw-Hill-Irwin. México
Chase, R.; Aquilano, N. Y Jacobs, R. (2009). Administración de operaciones "producción y cadena de suministros". ( $12^{\circ}$ ed.). Editorial McGraw-Hill-Irwin. México

David, F. (2008). Conceptos de Administración Estratégica. 6ta edición. Editorial Prentice Hall. México

Krajewski, L. y Ritzman, L. (2008). Administración de operaciones: estrategia y análisis. $7 \mathrm{ma}$ edición. Editorial Pearson Educación, S.A. México

López, S. (2008). La efectividad gerencial. Editorial Casa Hermanos. Venezuela

Muñoz, N. (2009). Administración de operaciones. Cengage Learning Editores. México

Nahmias, S. (2007). Análisis de la producción y las operaciones. Compañía Editorial Continental, S. A. México

Rodríguez, M. (2008). Diplomado en habilidades de gestión directiva para la competitividad y eficiencia institucional. Centro de Capacitación y Calidad, Jalisco. IMSS-SNTSS. Compilación de material y facilitadora del curso. México

Sallenave, J. (2009). Gerencia y planeación estratégica. Editorial Norma. Colombia

Schroeder, R. (2007). Administración de producción y operaciones. Cuarta Edición. Editorial Thompson. México

Skinner, W. (2010). Administración estratégica de operaciones. McGraw-Hill. México.

Slack, N., Chambers, S., y Johnston, R. (2007). Gerencia de Operaciones. 6ta edición. Editorial Mc Graw Hill. México

Wheelen, T. y Hunger, J. (2007). Administración estratégica y política de negocios. Décima edición. Editorial Pearson Educación, S.A. México 